\title{
Dissemination and Governance of Historical Nihilism in the Context of Big Data
}

\begin{abstract}
Weng Zhe
China Jiliang University School of Marxism Studies, Qian Tang, Hang Zhou, Zhe Jiang, China

550113639@qq.com

ABSTRACT

As a powerful information means, big data technology has the characteristics of strong permeability, wide coverage and high concealment. With the gradual deepening of the deconstruction of historical nihilism by mainstream ideology, historical nihilism has gradually shifted its main position from academic field to life field, offline to online, from researchers to netizens, and evolved new communication characteristics through big data, resulting in the erosion of mainstream ideology There are three major hazards: the pollution of the network public opinion environment and the misleading of netizens' historical cognition. In view of this, this paper will focus on the interaction between the three variables of technological progress, morphological evolution and governance change, and build an integrated theoretical model combined with the general political system theory. Based on the big data public opinion governance paradigm of "co construction, CO governance and sharing", improve the supervision system of big data platform, create a scientific and dialectical dialogue environment, and cultivate network citizens who know history and reason.
\end{abstract}

Keywords: internet, Historical nihilism, New form, Countermeasures.

\section{大数据背景下历史虚无主义的传播与治理}

\section{翁 哲}

中国计量大学马克思主义学院, 钱塘, 杭州, 浙江, 中国 550113639@qq.com

\section{摘要}

大数据技术作为一种强有力的信息化手段, 具有渗透性强、覆盖面广、隐匿度高的特性。随着主流意识形态对 历史虚无主义的解构逐步加深, 历史虚无主义也逐渐将主阵地由学术领域转向生活领域, 线下转向线上, 从研 究学者转向广大网民, 通过大数据嬗变出新的传播特征, 产生对主流意识形态的侵蚀、对网络與论环境的污染 和对网民历史认知的误导三大危害。鉴于此，本文将重点关注技术进步，形态嬗变、治理变革三个变量之间的 相互影响, 结合一般政治系统理论, 构建一个整合性理论模型。基于 “共建共治共享” 的大数据與情治理范式, 完善大数据平台的监管制度、营造科学辩证的对话环境、培养知史明理的网络公民。

关键词: 互联网; 历史虚无主义；新形态；应对措施

\section{1. 前言}

当今世界格局正逐渐走向多极化, 思想政治领域 的斗争也愈演愈烈。大数据自引入以来, 中国生产力 和生产关系发生了质的变化。彼时的接纳使得中国的 互联网产业在短时间内得到了迅速发展。另一方面则 便利了错误思潮的传播, 对主流意识形态构成冲击,
进而污化党的领导形象。在对历史本质的问题上，习 近平曾言: “历史就是历史, 事实就是事实, 任何人 都不可能改变历史和事实”。 ${ }^{[1]}$ 鉴于此, 我们必须深 入探究在大数据背景下历史虚无主义传播的新特点, 分析其危害, 并有效地进行抵御和防范。 


\section{2. 历史虚无主义大数据平台传播的主要特征}

近年来，为了在主流意识形态传播力不断增强的 大背景下谋得生存, 历史虚无主义也改变了其发展方 略, 在线上开疆扩土。其手段也适时而变, 从大数据 平台的特性出发, 通过向人们 “灌输虚无” 到引起人 们 “自发虚无”。阐释更为详细一些, 就是利用互联 网所营造的多方面信息交互大环境, 给群众灌输历史 虚无主义，使其不加具体分析而盲目否定人类社会的 历史发展过程，甚至否定历史文化，否定民族文化、 民族传统、民族精神，否定一切的历史观点和思想倾 向 ${ }^{[2]}$ 。相比以往, 此种手段渗透较为深入、行动较为 隐蔽、危害较为严重, 反制也更为困难。

\section{1. 隐匿性：主体角色模糊化}

在大数据视域下，历史虚无主义的传播逐渐趋向 隐蔽, 此类人群往往在普通民众、追随者、指引者、 灌输者和号召者等网络角色之间变换, 盅惑其他人为 自己发声。在以往互联网还未普及之时, 历史虚无主 义者的身份尚且还能较为容易地界定下来, 比如一篇 文章的作者或是一场讲座的报告人, 此类情形下, 主 体传播的效果仅仅针对于数量有限的读者和听者, 而 且容易受到與论的批判甚至是法律的制裁。

当前利用大数据以及算法对线上环境的全面监 测只是相对意义上的, 这无疑给历史虚无主义的传播 提供了可乘之机, 各类社交媒体成了滋养历史虚无主 义者的温床。首先, 网上传播权和选择权的自由化, 有一个重要的前提, 即网上传播者和接收者都能够采 取各种手段绕过大数据的监测, 隐匿真实身份, 以一 个或多个化名在网上出现。一方面虽然可以为网民的 传播活动提供安全保障, 另一方面却为历史虚无主义 的传播提供了保护壳。其次，在大数据视域下，思想 灌输的主体与客体互动性不断加强, 这给予了网民低 门槛的交互条件与平等的对话地位, 使其能够便捷地 进行讨论, 反应自己的思想、情感和诉求, 更可以利 用网络自身强大的技术优势, 将历史虚无主义思想灌 输的主体与客体置于同一时空之中, 进行点对点、点 对面、甚至面对面的实时交流与平等互动。历史虚无 主义传播者既能够发起一项话题, 也可以作为一个话 题的参与者, 前者能够使自己作为意见领袖引导话 题, 后者又能够作为从众者壮大其声势。一些世界观、 价值观未完全成熟的网民长期接触此类信息, 就会对 历史产生曲解，价值观产生倾斜。

\section{2. 对等性：传播手法横向化}

历史虚无主义在利用网络传播的过程中，传播路 径也不再采取传统的 “教育灌输” 手段, 而是转变成 了由内到外的引起 “共情” 的方法。在以往的历史虚 无主义的传播之中，历史虚无主义者格外注重 “主体 直接在场” 的效应, 使客体直接感受到主体的人格与 情感的力量, 主客体之间地位明显, 易于辨别。此种
手段无疑对时间、空间以及对象群体有着较高的要 求，而且面对当下取证手段日益丰富的实际，对于传 播者来说, 无疑存在着巨大的风险。

因而, 历史虚无主义的传播逐渐趋向于依托大数 据, 将主客体之间的身份相交融, 使双方以平等的身 份展开活动从而引起 “共识”。碎片化是当前网络信 息交互的一个重要特点, 平台对数据的判断和归纳也 有一定几率影响网络與情的体现, 网民信息获取的此 项特性和平台存在的缺陷受到利用, 使人们在一个轻 松愉快的氛围之中接受思潮潜移默化的影响。主体在 这个过程中隐晦地提出他们的观点, 并且采用一些文 字的暗示, 引导客体的思维走向, 从而达成自己的目 标, 大致地完成了历史虚无主义传播的流程。在这一 流程中, 参与者的身份平等, 看似仅仅是一群拥有自 己独特看法的网民, 主体的客观动机与主观臆断隐匿 于互联网平台这件外衣之下。广大网民由于缺乏历史 常识的历史判断能力, 在通过算法, 有指向性地提供 迷惑性思想观念交流中，主动地接受了历史虚无主义 的灌输, 内化成为自己有所偏差的历史见解, 从而将 身份由客体转变成新的主体, 进行新一轮的传播。

\section{3. 广泛性：传播范围扩大化}

随着中国互联网的不断发展，人民与大数据之间 的联系也变得日益密切了起来。当今的人们不单单只 能通过 PC 来进行网络社交, 智能终端和无线网络的 普及使得网民群体迅速扩大。从马克思主义的量变质 变规律来看, 当网民的数量规模达到一定的量级时, 会使得互联网的传播方式发生质的变化，从而使得普 及速度相较于以前迅速加快。相较于 2019 年的 8.5 亿网民规模，2020 年上半年中国网民规模达到了 9.4 亿, 增加了 9000 万。网民规模的扩大，意味着此间 未受系统历史教育的人群数量也在不断增多, 互联网 舆论事件对社会的渗透力和影响力也在逐渐加大加 深。在早期, 一个社会事件网络互动数超过十万, 就 会被认定是极其重要的舆论情况, 现如今, 随着微博 微信等社交平台的普及和网民数量的迅速增加, 在大 数据的加持下, 流量超百万级别的网络與论事件也屡 见不鲜。因而, 历史虚无主义者通常在广大网民群体 中，以“民主” “自由” “人权” “常识”为主线逻 辑, 绕过大数据平台的监测, 不顾前因后果地随意编 造篡改历史情节, 在网民中输出自己的立场, 毫无疑 问, 大数据的发展使得对象范围扩大, 受影响的群体 增多，手段更具针对性。

\section{3. 大数据视域下历史虚无主义传播的危害}

否定人民群众在历史进程中所发挥的决定性作 用, 对主流意识形态进行污化。历史虚无主义者们热 衷于利用大数据的交互功能和覆盖渗透功能, 营造出 一种心理与科技相结合的环境。在此种环境之下, 议 题大多摆着话题讨论或是事件评价的噱头, 真假难 辨, 极具诱导性, 从而改变个体对于历史事件的价值 
判断。

\section{1. 侵蚀主流意识形态, 弱化党的领导地位}

大数据时代, “流量” 的地位变得愈发重要。网 络话题的影响力和参与其中的用户数量呈正相关, 而 往往越是颠覆人们既定认知的事物, 越能够吸引人们 的眼球。诸如 “革命容易使人发疯发狂, 丧失理性。” “革命残忍黑暗肮脏的一面我们注意得很不够。”抓 住 “流血” 二字不放, 把共产党人近代以来的艰苦斗 争说成是血腥污秽的历史, 对腐朽政权仍保有幻想, 将其旧思想, 旧观念奉为圭香并向外灌输。在一些网 民的潜意识中, 原本就不牢固的历史认识根基也因此 动摇。产生的结果就是只关心历史事件的表象, 不去 探析其背后的深层原因, 否定人民群众在历史进程中 的决定性作用, 否定马克思主义的指导地位。

在马克思主义与其它思想观念的斗争过程中, 其 中所包含的历史唯物主义不可避免地成为了众矢之 的, 被扣上经济决定论之流的莫须有 “罪名” 数不胜 数。网络空间也概莫能外, 批评者们通过大数据平台 对数据的采集整理和运输特性聚集在一起, 针对历史 唯物主义关于历史规律性的观点进行言论输出, 从而 对马克思主义为主导的主流意识形态进行侵蚀。

\section{2. 污染线上对话环境, 形成不良社会风气}

以历史虚无主义为主的思想灌输, 流毒于依托大 数据搭建的社群、超级话题、公众账号、短视频等各 类虚拟场域之中。为借助大数据手段, 扩大历史虚无 主义的影响, 一些 “意见领袖” 建立起了以微信、微 博、抖音等主流社交媒体为要素的信息传播矩阵。其 不遗余力地提高自身在网络上的号召力的做法, 不仅 对网络與情的治理造成了巨大挑战, 也在社会上形成 了虚无历史的不良风气。

首先, “双微一抖”之类的社交平台用户基数大, 信息承载媒介大同小异, 历史虚无主义者此类平台的 主要特性, 首先是将文字音画等各项多媒体信息混合 加工, 营造出一个立体的信息传播环境, 给个体以更 加强烈的感官冲击, 从而增强其灌输效果。其次, 此 类平台往往开放评论, 使个体能够自由发表对某一特 定历史人物或历史事件的观点, 从运行的机制上赋予 了个体之间历史叙事的开放解释权, 网民们的从众心 理被利用。例如当某一主体在发表议题时, 为增强其 说服力和话题热度, 会事先与其他历史虚无主义者进 行沟通, 或是购买 “水军”, 在评论区营造一种该观 点是 “时下热门”、“众望所归” 的假象, 而大数据 对此种情形的监测往往不够全面, 久而久之, 在感官 刺激和开放解释权的感染下, 一些网民的历史认识和 政治观念偏离中心, 给社会带来了诸多不稳定因素, 增加社会风险。

\section{3. 扰乱网民历史认知, 造成人们思想混乱}

随着传播对象全民化程度加深, 其传播手段也从 灌输变为引导、诱导, 即目的在于加深和巩固客体与 主流意识形态相违背的错误思想, 引导其渐渐以感性 认识代替理性认识, 弃绝历史唯物主义的辩证性、严 密性和批判性。传播者所追求的不再是简单地在客体 头脑中植入历史虚无主义观点, 而是促使其以自我意 识掩盖整体格局, 从当下环境和利已主义价值观去重 述历史人物和历史事件。最终推动其以自发性传递代 替被动性接受, 传播客体在此过程中逐渐演变为主 体, 导致错误历史虚无主义的扩大化和深入化传播。

从表面上看, 在这一过程中, 参与者之间的地位 平等, 信息交互的形式也呈现多元化、开放化的特性, 某一特定观点会在与其他类似的观点的碰撞交融中 逐渐同化, 从而成为汇聚群体观点的集体共识。实则 从头至尾都穿插着历史虚无主义者的不着痕迹的诱 导和盅惑, 此机制给客体以足够的空间进行历史议题 的发表和对历史事件或历史人物的评论, 主观臆断愈 发占据主导地位, 理性、客观、整体、逻辑、规律等 视角在此过程中也逐渐地被淡化。此外, 看似人人都 能够通过大数据及时获取自己感兴趣的资讯, 而事实 上，这使得网民们所接受的信息越来越同质化，观 念中原本就错误的历史认知愈发根深蒂固了。

\section{4. 大数据时代历史虚无主义的治理}

思想领域如果被不具有科学真理性的主流价值 观去占领, 那么便会杂草丛生。历史虚无主义不仅引 导个体为其发声, 而且将错误的历史观点植入个体的 观念, 扭曲其思想, 冲击社会主义核心价值观。在采 取的各项治理措施中, 最常见的是重述史实。单纯的 历史叙事在与历史虚无主义传播者的隐匿、渗透、内 化等手段的应对中显得苍白无力。习总书记在十九大 报告指出: “要以培养担当民族复兴大任的时代新人 为着眼点, 强化教育引导、实践养成、制度保障, 把 社会主义核心价值观融入社会发展各方面, 转化为人 们的情感认同和行为习惯。

\section{1. 加强对平台的监管力度}

在大数据视域下, 对于算法和数据的掌握令企业 实现了某种意义上的自我确权, 而在一定程度上剥夺 了政府 “授权” 的权力一一这构成了最根本的统治风 险和最现实的治理挑战。 ${ }^{[4]}$ 有些历史虚无主义者正是 抓住了政商权力范围的真空部分作为自己的庇护, 一 方面隐匿其自身, 在法律法规未作明确规定的边缘地 带大打“擦边球”。另一方面营造出一种历史对话“无 人监管” 的假象, 使得 “言论自由” 的定义在广大网 民心目中被曲解。为此, 必须构建起完善的法律体系, 加强对大数据平台的监管与治理力度。

首先, 立改废释四项措施同步推进, 通过此类措 施使法律法规向网络空间延伸, 增强网络空间管理的 
强制性, 极为必要。以法为载体, 将外在的强制力与 内在的强制力结合起来, 引导个体在党纪国法的规范 和唯物史观的指导下进行历史对话，同时使历史虚无 主义传播者得到应有的惩罚。其次，加强对于短视频、 公众号平台、兴趣社区等新媒体业态和大数据推送、 ATL 等新技术应用的规范管理办法，对传播历史虚无 主义思潮后果做出明确规定，通过从根源入手，阻断 历史虚无主义的传播。“法与时转则治，治与时宜则 有功”, 加强大数据平台的法制建设, 有利于规整资 讯流传的秩序，确保国家安全、社会稳定。

\section{2. 营造科学辩证的对话环境}

随着社会思潮的影响力和传播范围逐渐扩大，社 会與论环境面临的风险与挑战逐渐增强, 大数据通过 增强信息辨别能力来营造科学辩证的历史对话环境 变得愈发重要。正向环境的建设，离不开多方的共同 作用。首先，历史虚无主义的面纱，必须要由事实去 揭开，对于一些指向历史人物或历史事件的质疑和谬 论，应该直接回应，以可靠的事实进行对话，在网民 观念中灌输贴合实际的历史事实和价值判断, 筑牢全 网历史记忆的根基, 抵御历史虚无主义的侵袭。其次, 在大数据视域下, 主流意识形态不可避免地会遭遇侵 蚀, 因此必须坚持历史唯物主义在网络历史对话环境 中的指导地位, 坚持社会主义核心价值观在网络环境 中的引领地位。综合利用各种载体, 提升正面信息的 趣味性, 深入加强四史教育, 通过富有亲和力和说服 力的语态语势解读党史、新中国史、改革开放史、社 会主义发展史, 深入浅出地解析历史虚无主义的危 害。最后, 互联网公司应自觉承担起社会责任, 要做 到 “与时俱进”、“因势利导” , 积极主动配合监管 部门采取的各项举措, 运用自家平台强大的影响力和 控制力, 以先进的互联网技术和监管理念, 塑造好、 管理好时下历史对话的环境。“兼听则明, 偏听则暗”, 这也正是营造科学辩证的网络对话环境所应坚持的 方向。

\section{3. 培养知史明理的网络公民}

马克思主义的鲜明立场在于以人民为中心，在与 历史虚无主义作斗争的过程中, 也应坚持一切为了人 民, 一切依靠人民。网民是社交平台最基本的组成部 分, 要重视培养广大民众最基本的历史辨别能力。首 先, 网络个体应当自觉提高历史文化素养, 尊重、学 习和敬畏历史, 充分接触信史、明史, 知史爱国, 做 到 “以良史之忧忧天下” ${ }^{[5]}$, 从而以自身的文化积累 来抵御历史虚无主义的侵蚀。其次, 网民的眼睛要做 到 “风吹不昏”、“沙打不迷”。“在分析任何一个 社会问题时, 马克思主义理论的绝对要求, 就是要把 问题提到一定的历史范围之内” ${ }^{[6]}$ 。用唯物史观武装 头脑, 指导实践, 在思想上牢牢占据反对历史虚无主 义的制高点, 将臆造的 “历史片段” 放进历史的逻辑 和规律中去检验, 揭穿其伪善的面具。最后, 网民在 对历史事件或历史人物进行认识的过程中，应注重线
上与线下相结合，根据信息接受方式的变化、生活场 域的变换，进行合理利用与有机组合，通过搜集资料 与实地参观等方式，在充分了解多手史料的同时，感 受到中华历史之源远流长, 从而不断传播文化自信, 更大程度地压缩历史虚无主义 “自由发挥” 的空间。

\section{5. 结论}

总的来说，大数据技术的普及和应用，增强了舆 情获取与监测能力, 为网络环境治理提供了新的途 径。然而，其带来的负面作用仍不可忽视，历史虚无 主义得以冒头，由于存在线上用户的隐匿性、信息交 互的对等性、网民的广泛性，历史虚无主义得以冒头， 乘着大数据的 “东风”, 在网民群体中扩大影响, 给 历史虚无主义思潮防范与治理工作带来新的风险与 挑战。因此, 我们需要健全对大数据平台的监督机制, 增强网络参与者的伦理自律意识, 培养知史明理的网 络公民，以史为鉴，开创未来。

\section{REFERENCES}

[1] Xi, J. (2014) Speech at the Ceremony Commemorating the Seventy-seventh Anniversary of the Outbreak of the National War of Resistance, 08: 20-21.

[2] Zhang, S, Yang, Y. (2000) New Terminology Dictionary Since the Third Plenary Session of the Eleventh Central Committee of the Party, Jinan Publishing, Jinan

[3] Xi, J. (2017) To Win the Great Victory of Socialism with Chinese Characteristics in the New Era By Building a Well-off Society in an All-round Way--Report on the 19th National Congress of the Communist Party of China. J. Party Construction, 11: 15-34.

[4] Peng, B. Zhang, Q. (2020) The Formation and Evolution of Internet Governance Mode in China (1994-2019). J. Journalism and Communication Research, 08:44-65+127.

[5] Gon, Z. (1975) Gong Zizhen Complete Works-New 1st Edition.Shanghai People's Publishing, Shanghai

[6] Lenin. (2012) Lenin Collection. Volume 2. People's Publishing, Beijing. 